\title{
The Theory Graph Modeling and Programming Systems from Module Elements to the Application Areas
}

\author{
E. M. Lavrischeva ${ }^{1}$ \\ ${ }^{1}$ Doctor of phys.-mat.Sci., Professor of MIPT, General Sci. Specialist ISPRAS, Russia \\ Correspondence: E. M. Lavrischeva, Doctor of phys.-mat.Sci., Professor of MIPT, General Sci. Specialist \\ ISPRAS, Russia.
}

Received: July 30, 2019

doi:10.5539/cis.v12n4p20

\author{
Accepted: September 10, 2019 \\ Online Published: September 24, 2019 \\ URL: https://doi.org/10.5539/cis.v12n4p20
}

\begin{abstract}
The mathematical basics of graph modeling and paradigm programming of applied systems (AS) are presented. The vertices of graph are been the functional elements of the systems and the arcs define the connections between them. The graph is represented by an adjacency and reach ability matrix. A number of graph of program structures and their representation by mathematical operations (unions, connections, differences, etc.) are shown. Given the characteristics of graph structures, complexes, units, and systems created from the modules of the graph. The method of modeling the system on the graph of modules, which describe in the programming languages (LP) and calling them with operations (link, assembling, building, etc.). The standard of configuration (2012) Assembly of heterogeneous software elements in AS of different fields of knowledge is made. Brief descriptions of modern and future programming paradigms for formal theoretical creation of systems from service-components for Internet in the near future are given.
\end{abstract}

Keywords: graph theory, adjacency matrix, reach ability, mathematical operations, configuration, Assembling, paradigm programming, future technologies

Mathematics is more than science,

It's the language of science.

Niles Bohr

\section{Introduction}

Programming theory is a mathematical science, the object of study of which is the mathematical abstraction of the functions of programs with a certain logical and information structure, focused on computer execution. With the advent of the LP began to develop new methods of analysis of algorithms of AS problems, the graph theory for the representation the structure AS by separate programs elements, displaying them in the vertices of the graph to create a complex structure of AS (programs, aggregate, complex, system, etc.). Programs elements of missile defense were first called modules, programs, then objects, components, services, etc. (Lavrischeva, 1982, 1991, 2014, 2016). For the formal specification of these elements were formed the corresponding programming paradigms, allowing from the point of view of the theory and graphs to describe the problems of different AS (medicine, biology, mathematic, chemistry, genetic, etc.).

\section{Graph Theory of Programs from Modules}

The basis for the creation of systems of modules was the method of assembling the graph (70-80 years of the last century) heterogeneous modules is implemented in the APROP system of IBM (Lavrischeva, 1982; Glushkov, 1976) and it was used to create specialized software and technical complexes of the MIC project as Assembly programming (Lavrischeva, 1991; Lipaev, 1992). This programming (Lavrischeva, 2009) as was said (Ershov, 1977) "provided the building is already existing individual pieces of software (reuses) in the complex structure systems". The interface of the modules was described initially in a special description language IDM (Interface Definition Language) for linking modules to the complex structure (Ekaterina, 2016). After 1992 there were new languages for linking and interaction of different language modules into complex systems (IDL, API, WSDL, etc) for linking modules as on fabrics programs (Ekaterina, 2016) and the statement Configuration (config) in the standard IEEE 828-2012 for receiving a configuration file of any AS from modules for AS (http://www.scilogs.com/scientific_and_medical_libraries). 
A module is a formally described program element that displays certain AS function that has the property of completeness and connectivity with other elements according to the data specified in the interface part of the description. From a mathematical point of view, a module is a mapping of a set of initial data $\mathrm{X}$ to a set of output $\mathrm{Y}$ in the form $\quad M: X \rightarrow Y$.

A number of restrictions and conditions are imposed on $X, Y$ and $M$ to make the module an independent program element among other types of program objects (Lavrischeva, 1991, 2009).

Types of connections between modules via input and output parameters are as follows:

1) linking of control: $C P=K_{l}+K_{2}$, where $K_{1}$ is the coefficient of the calling mechanism; $K_{2}$ is the coefficient of transition from the environment of the calling module to the environment of the called;

2) linking of data $C I=\sum_{i=1} K_{i} F\left(x_{i}\right)$, where $K_{\mathrm{i}}$ - the weight coefficient $i$ - parameter;

$F\left(x_{i}\right)$ - the function of the parameter $x_{i}$. Coefficients $K_{i}=1$ - for simple variables and $K_{i}>1$ for complex variables (array, record, etc.). $F\left(x_{i}\right)=1$ if $x_{i}$ - a simple variable and $F\left(x_{i}\right)>1$ if complex variable.

The program of modular structure is given by the graph $G=(X, E)$, where $X$ - a finite set of vertices; $E$ - a finite subset of the direct product of $X \times X \times Z$ the set of relations on the arcs of the graph. The program structure represents a pair $S=(T, \chi)$, where $T$ - a model of a program of modular structure; $\chi$ - a characteristic function given on the set of vertices $X$ of the graph $G$.

The value of the characteristic function $\chi$ is defined as:

$$
\begin{aligned}
& \chi(\mathrm{x})=1 \text { if the module with vertex } \mathrm{x} \in \mathrm{X} \text { is included in the modular system; } \\
& \chi(\mathrm{x})=0 \text { if the module with vertex } \mathrm{x} \in \mathrm{X} \text { is not included in the modular system and is not } \\
& \text { accessed from other modules. }
\end{aligned}
$$

Definition 1. Two models of program structures $T_{1}=\left(G_{1}, Y_{1}, F_{1}\right)$ and $T_{2}=\left(G_{2}, Y_{2}, F_{2}\right)$ are identical if $G_{1}=G_{2}, Y_{1}$ $=Y_{2}, F_{1}=F_{2}$. The $T_{1}$ model is isomorphic to the $T_{2}$ model if $G_{1}=G_{2}$ between sets $Y_{1}$ and $Y_{2}$ exists an isomorphism $\varphi$ and for any $\mathrm{x} \in \mathrm{X}, \mathrm{F}_{2}(\mathrm{x})=\varphi\left(\mathrm{f}_{1}(\mathrm{x})\right)$.

Definition 2. Two program structure $S_{1}=\left(T_{1}, \chi_{1}\right)$ and $S_{2}=\left(T_{2}, \chi_{2}\right)$ are identical if $T_{1}=T_{2}, \chi_{1}=\chi_{2}$ and the structures $S_{1}$ and $S_{2}$ are isomorphic, then $T_{1}$ is isomorphic to $T_{2}$ and $\chi_{1}=\chi_{2}$.

The concept of isomorphism of program structures and their models is used in the specification of the abstraction level at which operations on these structures are defined. For isomorphic graph objects, operations will be interpreted in the same way without orientation to a specific composition of program elements, provided that such operations are defined over pairs $(\mathrm{G}, \chi)$. The software module is described in the LP and has an interface section in which external and internal parameters are set for data exchange between related modules through Call/RMI.

The interface defines the connection of heterogeneous software modules according to the data and the way they are displayed by programming systems with the $L P$. Its main functions are data transfer between program modules and their conversion to the equivalent form and transition from the environment and platform of the called module and back. Functions of conversion of non-equivalent data types is carried out with the help of a previously developed library of 64 primitive functions for heterogeneous data types of $L P$ in the APROP system (Glushkov, 1976; Lavrischeva, 2009, 27 December 2016) and included in the common system environments of (IBM, MS, Oberon, UNIX, etc.).

In this article we consider the mathematical theory of graphs of software modular structures and mathematical operations (union, projection, difference, etc.) implementation of linking the graph modules and the semantics of the data transformation for Big Data (Lavrischeva, 27December 2016) by the vertices of the module graph. Now software modules are described in modern LP (C, C++, Python, Java, etc) and assembling with help of the new paradigms programming (Lavrischeva, 2016 OCM, 2017 Yurait; Bruno, 2016).

\subsection{The Graph Modular Programs Structure of Definition}

To represent modular structures, we use the mathematical apparatus of graph theory, in which the graph $G$ is treated as a pair of objects $G=(X, E)$, where $X$ - a finite set of vertices, and $\Gamma$ is a finite subset of the direct product of $\mathrm{X} \times \mathrm{X} \times \mathrm{Z}$ - arcs of the graph, corresponding to a finite vertex (Fig. 1).

Definition 3. A program aggregate is a pair $S=(T, \chi)$, where $T$ - a model of the program modular structure of the 
aggregate; $\chi$ - a characteristic function defined on the set of vertices $\mathrm{X}$ of the graph of the modular structure $\mathrm{G}$. The value of the $\chi$ function is defined as follows:

$$
\begin{aligned}
& \chi(\mathrm{x})=1 \text { if the module corresponding to the vertex } \mathrm{x} \in \mathrm{X}, \text { - included in the unit; } \\
& \chi(\mathrm{x})=0 \text { if the module corresponding to the vertex } \mathrm{x} \in \mathrm{X} \text {, - not included in the software unit, but it }
\end{aligned}
$$
is accessed from other modules previously included.

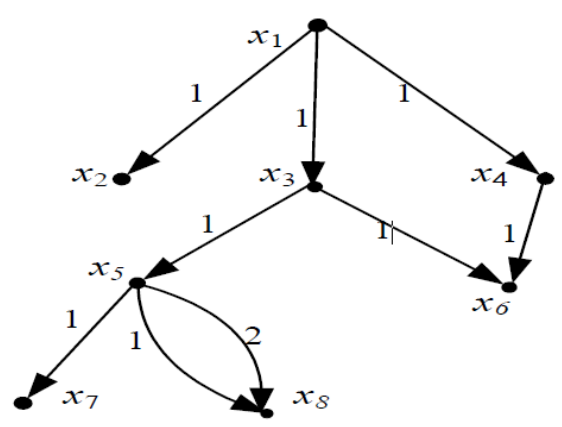

Figure 1. Graph from modules

The set of arcs of the graph have the form: $\mathrm{E}=\{(\mathrm{x} 1, \mathrm{x} 2,1),(\mathrm{xl}, \mathrm{x} 3,1),(\mathrm{x} 5, \mathrm{x} 8,1),(\mathrm{x} 5, \mathrm{x} 8,2)\}$. Based on this definition, we can say that the graph $\mathrm{G}$ is a multi-graph, since its two vertices can be connected by several arcs. To distinguish these arcs introduced their numbering positive integers - 1, 2. (Fig.1) and vertices of the graph $x 1$, $\mathrm{x} 2, \ldots, \mathrm{x} 8$ form a set of $\mathrm{X}$. From the module corresponding to the vertex $\mathrm{x} 5$, there are two link to the modules, with vertices $\mathrm{x} 7, \mathrm{x} 8$.

Definition 4 . The model of the program structure of the program unit is an object described by the triple $T=(G$, $\mathrm{Y}, \mathrm{F})$, where $\mathrm{G}=(\mathrm{X}, \mathrm{E})$ - a directed graph of a modular structure;

$\mathrm{Y}$ is a set of modules included in the program aggregate;

$\mathrm{F}$ is a correspondence function that puts an element of the set $\mathrm{y}$ at each vertex $\mathrm{X}$ of the graph.

$$
\text { Function F maps } \mathrm{X} \text { to } \mathrm{Y}, \quad \mathrm{F}: \mathrm{X} \rightarrow \mathrm{Y} \text {. }
$$

In General, an element from $\mathrm{Y}$ can correspond to several vertices from the set $\mathrm{X}$, which is typical for the dynamic structure of the aggregate (Lavrischeva, 2009, 2014).

The graph of software aggregates has the following properties:

1) graph $G$ has one or more connectivity elements, each of which represents an acyclic graph, i.e. does not contain oriented cycles;

2) in each graph $G$ is allocated a single vertex, which is called the root and is characterized by the fact that there are no arcs included in it and the corresponding module of the software unit is performed first;

3) cycles are allowed only for the case when some vertex has a recursive reference to itself. Typically, this feature is implemented by the compiler with the corresponding LP and this type of communication is not considered by the intermodule interface. Therefore, such arcs are not included in the graph. The exception to the consideration of other types of cycles is due to the fact that some modules will have to remember the history of their calls in order to return control correctly, which contradicts the properties of the modules;

4) an empty graph G0 corresponds to an empty program structure.

Next, the graph $\mathrm{G}$ will be used to illustrate mathematical operations on modular structures. In Figure 2, three types of subgraphs are shown and their description is given.
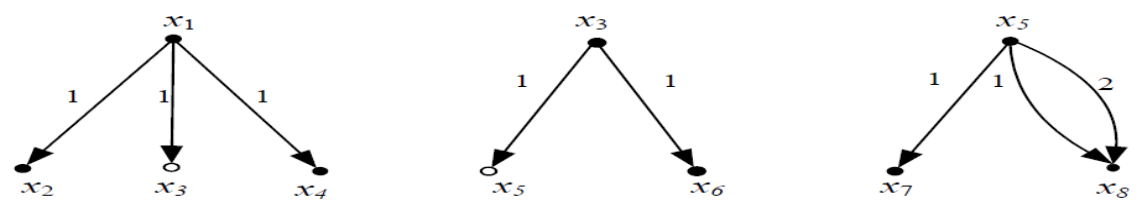

Figure 2. The graphs of modules structures $x_{1}, x_{3}, x_{5}$ 
A subgraph - a fragment of a software aggregate $G^{s}=\left(X^{s}, E^{s}\right)$ for whose functions one of two conditions $\mathrm{S}$ is satisfied:

$C(S)=1$, if $\chi(\mathrm{x})=1$ for any vertex $x$ of $X$;

$C(S)=0, \quad$ if there is $\mathrm{x}$ such that $\chi(\mathrm{x})=0$;

$R\left(S^{S}\right)=0$, if the modular structure is part of a higher-level structure and $R(S)=1$ if the software assembly is ready to run.

Given these combinations $\mathrm{C}$ and $\mathrm{R}$, the subgraph can be: open $(C=0, R=0)$; closed at the top $(C=0, R=1)$; closed at the bottom $(C=1, R=0)$.

The graph of the module is represented as $\mathrm{G}^{\mathrm{m}}=\left(X^{m}, E^{m}\right)$. It contains a single vertex $\mathrm{x} \in X^{m} \quad$ for which $\chi\left(\mathrm{x}_{\mathrm{j}}\right)=1$. This vertex is the root. An arc of the form $\left(x_{j}, x_{e}, k\right)$ means calling the module to the corresponding vertex $x_{j}$, i.e. to the module with the vertex $\mathrm{x}_{1}$. The dark circle on the graph corresponds to the vertex for which $\chi(\mathrm{x})=1$; light $-\chi(\mathrm{x})=0$.

Program graph $\mathrm{G}^{\mathrm{p}}=\left(X^{p}, E^{p}\right)$ which is performed $C\left(S^{p}\right)=1 ; \mathrm{R}\left(S^{p}\right)=1$. An example of a graph of such a program modular structure is shown in Fig. 1.

The graph of the complex $G^{c}=\left(X^{c}, E^{c}\right)$ consists of n connectivity components $(\mathrm{n}>1)$, each of which is a graph and includes: $\quad \mathrm{G}^{\mathrm{c}}=G_{1}^{p} \cup G_{2}^{p} \cup, \ldots, \cup G_{n}^{p}$,

where $X^{c}=X_{1}^{p} \bigcup X_{2}^{p} \bigcup, \ldots, \bigcup X_{n}^{p}$ и $E^{c}=E^{p} E_{1}^{p} \cup E_{1}^{p} E_{2}^{p} \cup, \ldots, \cup E_{n}^{p}$.

These definitions of the graph of the program module, program, aggregate and complex are used for the process of assembling the modules. These concepts may differ from similar ones, which are considered in other contexts of the work.

\subsection{Matrix Representation of the Graph from Program Elements of Module Type}

Graph theory is used in information technology (for example, in solving problems of control, communication, design of electrical circuits, programming of complex program structures, etc.). To compile an algorithm for solving problems on graph structures, reachability (incidence) and adjacency matrices are used. The incidence matrix is of size $\mathrm{nx} \mathrm{m}$, where $\mathrm{n}$ is the number of vertices of the graph, $\mathrm{m}$ is the number of edges of the graph. The matrix adjacencies correspond to vertices and columns to edges of the graph. The adjacency matrix allows establishing a set of vertices adjacent to a given vertex and is represented by a two - dimensional array of size $\mathrm{n}$ $\mathrm{x} \mathrm{n}$, where $\mathrm{n}$ is the number of vertices of the graph (Lavrischeva, 1991, 2009).

To determine the main operations on software structures, we use these mathematical apparatus of the matrix representation of graphs in the form of an adjacency and reachability matrix. That is, the graph (fig.1) is represented by the matrix $M=m(i, j)$ of adjacency and is proved by the reach ability matrix (Evstigneev, 1985; Lavrischeva, 2017; Theory Graphs, wiki). The element of the matrix $m_{i j}$ determines the number of call operators with index $i$, to the module with index $j$.

In addition to the adjacency matrix (calls), the characteristic vector $V i=\chi(x i)$ for $i$-element is used. For a modular structure graph (Fig. 1) characteristic vector and adjacency matrix have the form:

$$
V=\left(\begin{array}{l}
1 \\
1 \\
1 \\
1 \\
1 \\
1 \\
1 \\
1
\end{array}\right)
$$$$
M=\left(\begin{array}{llllllll}
0 & 1 & 1 & 1 & 0 & 0 & 0 & 0 \\
0 & 0 & 0 & 0 & 0 & 0 & 0 & 0 \\
0 & 0 & 0 & 0 & 1 & 1 & 0 & 0 \\
0 & 0 & 0 & 0 & 0 & 1 & 0 & 0 \\
0 & 0 & 0 & 0 & 0 & 0 & 1 & 2 \\
0 & 0 & 0 & 0 & 0 & 0 & 0 & 0 \\
0 & 0 & 0 & 0 & 0 & 0 & 0 & 0 \\
0 & 0 & 0 & 0 & 0 & 0 & 0 & 0
\end{array}\right)
$$ 
We analyze adjacency matrices and characteristic vectors for subgraphs and graphs of modular structures corresponding to different types - program, complex, aggregate, etc. For subgraphs (Fig.2) vectors and matrices have the form:

$$
\begin{gathered}
V_{3}^{s}=\left(\begin{array}{l}
1 \\
0 \\
0
\end{array}\right), \quad M_{3}^{s}=\left(\begin{array}{lll}
0 & 1 & 1 \\
0 & 0 & 0 \\
0 & 0 & 0
\end{array}\right) ; \\
M_{1}^{s}=\left(\begin{array}{cccc}
0 & 1 & 1 & 1 \\
0 & 0 & 0 & 0 \\
0 & 0 & 0 & 0 \\
0 & 0 & 0 & 0
\end{array}\right) ; \quad V_{5}^{s}=\left(\begin{array}{l}
1 \\
1 \\
1
\end{array}\right), \quad M_{5}^{s}=\left(\begin{array}{lll}
1 \\
1 \\
0 \\
0
\end{array}\right),
\end{gathered}
$$

For the program graph (Fig. 1) the characteristic vector and the matrix of calls coincide with $\mathrm{V}$ and $\mathrm{M}$, respectively, and determine the form (2), in which all elements of $\mathrm{V}$ are equal to one. In the case of the complex, the characteristic vector and the call matrix have the following form:

$$
V^{c}=\left(\begin{array}{c}
V_{1}^{p} \\
V_{2}^{p} \\
\ldots \\
V_{n}^{p}
\end{array}\right), \quad M^{c}=\left(\begin{array}{cccc}
M_{1}^{p} & 0 & \ldots & 0 \\
0 & M_{2}^{p} & \ldots & 0 \\
\ldots & \ldots & \ldots & \ldots \\
0 & 0 & \ldots & M_{n}^{p}
\end{array}\right)
$$

Here $V_{i}^{p}$ and $M_{i}^{p} \quad(i=\overline{1, \mathrm{n}})$ denote the characteristic vector and the adjacency matrix for the graph of the $i$-th program included in the graph of the complex. In the future, the matrix representation is used when performing mathematical operations on software structures.

\subsection{The Relation of the Reachability Graph of Program Structures}

Let $G=(X, E)$ - a graph of a program of modular structure; $x_{i}, x_{j}$ - vertices belonging to $X$. If there is an oriented chain from $x_{i}$ to $x_{j}$ in the graph $G$, then the vertex $x_{j}$ is reachable from the vertex $x_{i}$. The following statement is true: if the vertex $x_{j}$ is reachable from $x_{l}-$ из $x_{j}, x_{l}-$ from $x_{j}$, then $x_{l}$ is reachable from $x_{l}$. The proof of this fact is obvious (Lavrischeva, 2017 p.38-50).

Consider a binary relation on the set $\mathrm{X}$ that determines the reach ability of one vertex of a graph to another. We introduce the notation $x_{i} \rightarrow x_{j}$ - reach ability of the vertex $x_{j}$ from $x_{i}$. The relation is transitive. Denote by $\left.D\left(x_{i}\right)\right)$ the set of vertices of graph $\mathrm{G}$ reachable from $x_{i}$. Then the equality of determines the transitive closure of $x_{i}$ in relation to the achievability of tops. We prove the following theorems.

$$
\overline{x_{i}}=\left\{x_{i}\right\} \text { 茂婈 }\left(x_{i}\right) \text {; }
$$

Theorem 1. For the selected element of connectivity of the graph of the program structure, any vertex is reachable from the root corresponding to the given vertex of the graph, i.e. the equality ( $\mathrm{x}_{1}-$ root vertex)

$$
\overline{x_{1}}=\left\{x_{1}\right\} \text { 茂版 }\left(x_{1}\right)=X \text {. }
$$

Evidence. Suppose the vertex $x_{i}\left(x_{i} \in X\right)$ is unattainable from $x_{1}$. Then $x_{i} \notin \overline{\mathrm{X}_{1}}$ and the set $\mathrm{X}^{\prime}=X \backslash \overline{\mathrm{X}_{1}}$ - not empty. Since the selected component of the graph is connected, there is a vertex $x_{j} \in \overline{\mathrm{x}_{1}}$ and a chain $H\left(x_{i}, x j\right)$, leading from $x_{i}$ to $x_{j}$. Based on the acyclicity of the graph $\mathrm{G}$, in $X^{n}$ there should be a simple chain $H\left(x_{i}, x_{j}\right)$, where the vertex $x_{l}$ does not include arcs (this chain can be empty if $X^{\prime}$ consists only of $x_{i}$ ). Consider the chain $H\left(x_{l}, x_{j}\right)=$ $H\left(x_{l}, x_{i}\right) \mathrm{U} H\left(x_{i}, x_{j}\right)$. This means that the module $x_{i}$ is reachable from vertices $x_{l}$ and $x_{i}$ and both vertices contain no incoming arcs. This contradicts the definition of a graph of a modular structure with a single root vertex. 
The theorem is proved.

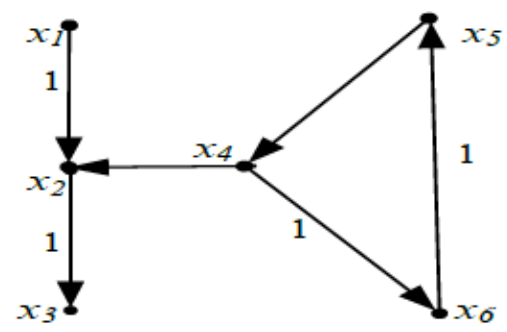

Figure 3. A graph that contain cycle

The results of this theorem are important to substantiate the requirement of the absence of oriented cycles in the graph of the program structure with respect to the notion of reachability. Consider the graph shown in Fig. 3 . From this figure it is clear that the graph contains a directed cycle and modules corresponding to vertices $\mathrm{x}_{4}, \mathrm{x}_{5}$, $\mathrm{x}_{6}$ will never be executed.

Thus, the results of theorem 1 reinforce the requirement that there are no oriented cycles in the graph of the program structure. We analyze the matrix representation of the reach ability relation for the graph of the program structure Fig.1 with the reach ability matrix A, which has the form (7). Coefficient aij = 1 if the module corresponding to the index 1 is reachable from the module corresponding to the index $i$ the Following results are based on the following theorem from graph theory.

$$
\mathbf{A}=\left(\begin{array}{cccccccc}
x_{1} & x_{2} & x_{3} & x_{4} & x_{5} & x_{6} & x_{7} & x_{8} \\
0 & 1 & 1 & 1 & 1 & 1 & 1 & 1 \\
0 & 0 & 0 & 0 & 0 & 0 & 0 & 0 \\
0 & 0 & 0 & 0 & 1 & 1 & 1 & 1 \\
0 & 0 & 0 & 0 & 0 & 1 & 0 & 0 \\
0 & 0 & 0 & 0 & 0 & 0 & 1 & 1 \\
0 & 0 & 0 & 0 & 0 & 0 & 0 & 0 \\
0 & 0 & 0 & 0 & 0 & 0 & 0 & 0 \\
0 & 0 & 0 & 0 & 0 & 0 & 0 & 0
\end{array}\right)
$$

Theorem 2. The coefficient $m_{i j}$ of the $l$-th degree of the adjacency matrix $M^{l}$ determines the number of different routes containing $l$ arcs and connecting vertex $\mathrm{x}_{\mathrm{i}}$ to the vertex of the $\mathrm{x}_{\mathrm{j}}$-oriented graph. The proof of this theorem is given in (Lavrischeva, 2017). Consider the following three consequences of this theorem.

Corollary 1.1. Matrix $\bar{M}=\sum_{1}^{n} M^{i}$, where $M$ is the adjacency matrix of a directed graph with n vertices coincides up to the numerical values of the coefficients with the reachability matrix $A$.

Evidence. In a directed graph containing $\mathrm{n}$ vertices, the maximum path length without repeating arcs cannot exceed $\mathrm{n}$. Therefore, the sequence of degrees of the adjacency matrix $M^{i}$, where $i=1,2, \ldots \ldots \mathrm{n}$ determines the number of all possible paths in the graph with the number of arcs $\leq \mathrm{p}$. Let the coefficient $m_{i i}$ of the matrix M be different from zero. This means that there is a degree of matrix $M^{i}$ in which the corresponding coefficient $\bar{m}_{i i}$ is also nonzero. Therefore, there is a path from vertex $\mathrm{x}_{\mathrm{i}}$ to $\mathrm{x}_{\mathrm{j}}$, i.e. vertex $\mathrm{x}_{\mathrm{j}}$ is reachable from $\mathrm{x}_{\mathrm{i}}$. This consequence determines the connection of the matrix of calls of the graph of the modular structure $M$, coinciding with the reachability matrix $\mathrm{A}$, and determines the algorithm for constructing the latter.

Corollary 1.2. Let there be a coefficient $m_{i i}>0$ for some $i$ in the sequence of degrees of the adjacency matrix $\mathrm{M}^{\mathrm{i}}$. Then there is a cycle in the original graph.

Evidence. Let $m_{i i}>0$ for some $l$. Therefore $x_{l}$ reachable from $x_{i}$, i.e. there is a cycle. According to the theorem, this cycle has $l$ arcs (generally repeated).

Corollary 1.3. Let the $n$-th degree of the adjacency matrix of the $M^{n}$ of the acyclic graph coincide with the zero matrix (all coefficients are zero). 
Evidence. If the graph is acyclic, then the simplest path cannot have more than $n-1 \operatorname{arcs}$.

If $M^{n}$ has a coefficient other than zero, then there must be a path consisting of $n$ arcs. And this way can only be oriented cycle. Therefore, all coefficients of $M^{n}$ for an acyclic graph are zero. This consequence provides a necessary and sufficient condition for the absence of cycles in the graph of a modular structure.

For acyclic graphs, the reachability ratio is equivalent to a partially strict order. The transitivity of the reachability ratio was considered above. Anti-symmetry follows from the absence of oriented cycles: if the vertex $x_{j}$ is reachable from $x_{j}$, then the opposite is not true.

We introduce the notation $x_{i}>x_{j}$ if vertex $x_{j}$ is reachable from vertex $x_{i}$.

Let $\mathrm{G}=(X, E)$ be an acyclic graph corresponding to some program structure.

Consider the decreasing chain of elements of a partially ordered set $\mathrm{X}: x_{i 1}>x_{i 2}>\ldots>x_{i n} \ldots \ldots$,

where " > " denotes the reachability ratio.

Since $\mathrm{X}$ is finite, the chain breaks. The verte $x_{i n}$ has no outgoing arcs, i.e. the element $x_{i n}$ is minimal (it corresponds to a module that does not contain access to other modules). The maximum element in the set $X$ is the root vertex.

\subsection{Mathematical Operations on the Graph Elements}

Mathematical operations $(\mathrm{U}, \cap, /,+,-, P, C, R)$ on graphs are performed at the level of abstractions of elements of program structures that lead to changes in graph elements and characteristic functions of systems: $\mathrm{S}=(\mathrm{G}, \chi)$ ( Lavrischeva, 2014, 2017 Yurait).

Let $S_{1}=\left(G_{1}, \chi_{1}\right)$ and $S_{2}=\left(G_{2}, \chi_{2}\right)$ be two graphs of program structures $G_{1}=\left(X_{1}, E_{1}\right)$ and $G_{2}=\left(X_{2}, E_{2}\right)$ respectively.

We introduce the following notations:

$$
\begin{aligned}
& D(x) \quad \text { - the set of vertices reachable from the vertex } \mathrm{x} ; \\
& D^{*}(x) \text { - the set of vertices from which vertex } \mathrm{x} \text { is reachable. }
\end{aligned}
$$

The same symbols are used for the same vertices included in the graphs $G_{l}$ and $G_{2}$. The main operations on the program structures are discussed below

Merge (join) operation $S=S_{1} \mathrm{U} S_{2}$

is intended to form a graph of the structure of the complex and is formally defined as follows $S_{1}$ and $S_{2}$ - any program structures that satisfy the definitions of claim 1:

$$
G=G_{1} \oplus G_{2}, \quad X=X_{1} \oplus X_{2}, \quad E_{1} \oplus E_{2},
$$

where the symbol denotes a direct sum provided:

$$
\begin{aligned}
& \chi(x)=\chi_{1}(x), \text { if } \chi \in X_{1}, \\
& \chi(x)=\chi_{2}(x), \text { if } \chi \in X_{2} .
\end{aligned}
$$

The same vertices included in $G_{1}$ and $G_{2}$ are represented by different objects in the operations of combining program structures. The characteristic vector and adjacency matrix of the program structure $\mathrm{S}$ are defined as follows:

$$
V_{l, 2}=\left(\begin{array}{l}
V_{1} \\
V_{2}
\end{array}\right), M_{l, 2}=\left(\begin{array}{cc}
M_{1} & 0 \\
0 & M_{2}
\end{array}\right),
$$

where $V_{1,2}$ and $M_{1,2}$ are characteristic vectors and adjacency matrices of modular structures $S_{1}$ and $S_{2}$ respectively. This operation is associative, but not commutative - the order of the operands determines the order of the components of the complex.

It should be noted that if the operands $S_{1}$ and $S_{2}$ satisfy the conditions for defining program structures, the result $S$ will also satisfy the same requirements. The join operation increases the number of connected graph elements. In addition, the column structures may themselves have multiple items of connectedness. For the rest of the operation counts of the operands and result are the only element of connection.

The connection operation. We denote by $x_{i}$ and $x_{j}$ the root vertices of graphs $G_{l}$ and $G_{2}$ of program structures $S_{l}$ and $S_{2}$, respectively. This operation $\mathrm{S}=S_{1}+S_{2}$, 
which is execute if these structures meet the following conditions:

$$
\begin{gathered}
\text { set } \mathrm{X}^{\prime}=X_{2} \cap X_{2} \text { not empty; } \\
\text { vertex } x_{j} \in \mathrm{X}^{\prime} \text { and } \chi\left(x_{j}\right)=0 ; \\
D^{*}(x) \cap D(x)=0 \text { for every } \quad x \in X^{\prime} \text {, where } D^{*}(x) \in X_{1} \quad \text { и } \quad D(x) \in X_{2} ; \\
G=G_{l} \mathrm{U} G_{2}, \quad X=X_{1} \mathrm{U} X_{2}, \quad E=E_{l} \mathrm{U} E_{2},
\end{gathered}
$$

The characteristic function $\chi$ is satisfied under the condition:

$$
\begin{gathered}
\chi(x)=\chi_{1}(x), \text { if } x \in X_{1} \backslash \mathrm{X}^{\prime} ; \\
X(x)=\max \left(\chi_{1}(x), \chi_{2}(x)\right)>\text { if } x \in X^{\prime}, \\
\chi(x)=\chi_{2}(x), \text { if } x \in X_{2} \backslash \mathbf{X}^{\prime} .
\end{gathered}
$$

First condition means that there are common vertices in graphs $G_{1}$ and $G_{2}$. According to the second condition, the root vertex $G_{2}$ belongs to the common part and for $S_{1}$ the object corresponding to $x_{j}$ is not included in the program structure yet.

The third condition prohibits the existence of cycles in the result graph. Indeed, if there is $x_{n} \in D^{*}(x) \cap D(x)$, then $x_{n}>x$ and $x>x_{n}$, and $x>x_{n}$, then this means the existence of a cycle.

If $S_{1}$ and $S_{2}$ satisfy the above conditions, the connection operation is partial.

Let us determine whether the result of the connection operation belongs to the class of program structures. Since $X^{\prime \prime}$ is not empty, the graph $G$ has one connected component. The root vertex of the graph $G$ is $\mathrm{x}_{\mathrm{i}}$. The graph $G$ itself has no oriented cycles, i.e. is acyclic.

Thus, $S$ belongs to the class of program structures under consideration.

This connection operation is not commutative and is generally not associative. To show that this operation is not associative, consider the result $S=\left(S_{1}+S_{2}\right)+S_{3}$, where the root vertices of graphs $\mathrm{G}_{2}$ and $\mathrm{G}_{3}$ are part of the vertices of graph $\mathrm{G}_{1}$ and $X_{2} \cap X_{3} \neq 0$.

Then the result of the $S_{2}+S_{3}$ join operation is undefined.

The operation of projection. Let $S_{l}=\left(G_{1}, \chi_{l}\right)$ be a program structure and $x_{i} \in X_{1}$. The operation of projection of this structure to the top of the graph $S_{1}$ is denoted as $S=P_{r x i}\left(S_{l}\right)$ and is defined as

$$
G(X, E), \quad X=\bar{x}_{i}, E=\left\{\left(x_{i}, x_{j}, K\right) \mid x_{i}, x_{j} \in X\right\},
$$

for the characteristic function is $\chi(x)=\chi_{I}(x)$, if $x \in X$. The projection operation defines the program structure $S_{I}$ in the structure $\mathrm{S}$. let's check the belonging of the structure $\mathrm{S}$ to the class of the considered program structures. If the graph of the structure $S_{1}$ is connected acyclically, then the same properties will be possessed by the graph G. There is a single root vertex $x_{i}$ in the graph $\mathrm{G}$. Thus, the program structure $S$ belongs to the class under consideration.

The difference operation for program structures is defined as follows. Let $S_{l}=\left(G_{l}, \chi_{l}\right)$ be a program structure and $x_{i} \in X_{1}$. The difference operation is performed on this structure and its projection to the vertex $x_{i}$ of the corresponding graph $\left(x_{i}\right.$ is not the cortical vertex of the graph $\left.G_{l}\right)$. Formally, the difference operation of the program structure has the form: $\quad S=S_{I}-P_{r_{x i}}\left(S_{I}\right)$,

and defined as follows: $\quad G=(X, E), \quad X=\left(X_{I} \backslash \overline{x_{i}}\right) \cup \mathrm{X}^{\prime}$

$$
E=\left\{\left(x_{i}, x_{j}, K\right) \mid x_{i}, x_{j} \in X\right\},
$$

where the set $X^{\prime}$ consists of such elements for which

$$
X^{\prime}=\left\{x_{j}^{\prime} \mid\left(x_{l} \in X_{l} \backslash x_{i}\right) \&\left(x_{j}^{\prime} \in \bar{x}_{i}\right) \&\left(x_{l}, x_{j}^{\prime}, K\right) \in E\right\}
$$


Here, the characteristic function $\chi$ is defined as:

$$
\begin{aligned}
& \chi(x)=\chi_{I}(x), \text { если } x \in X_{I} \backslash \overline{x_{i}} ; \\
& \chi(x)=0), \text { если } x \in X^{\prime} .
\end{aligned}
$$

The set $X$ includes vertices that are not included in the set $\bar{x}_{i}$, and those vertices $\bar{x}_{i}$ that include arcs from vertex $\mathrm{X}_{1} \backslash \overline{x_{i}} \quad\left(\right.$ sets $\left.X^{\prime}\right)$. The characteristic function for elements $x^{\prime} \in X^{\prime}$ is zero. The difference operation is the inverse of the join operation, i.e. the equality is performed: $S-P_{r_{x i}}(S)+P_{r_{x i}}(S)=S$.

Let us check that $S$, defined in (15), belongs to the class of program structures. If the graph is $G$, connected and acyclic, then the graph $G_{l}$ will have the same properties. The root vertex $G$ is the same as the root vertex $G_{l}$. Thus, $S$ satisfies the conditions for determining the program structure given in paragraph 1 .

Let $\mathrm{S}^{*}$ be the set of program structures given by the direct product $\mathrm{G}^{*} \mathrm{X} \chi^{*}$, where $G^{*}$ and $\chi^{*}$ are the set of graphs and the set of characteristic functions. Denote by $\Omega=\{\mathrm{U}, \cap, /,+,-\}$ - set of mathematical operations on program structures and $P, C$ and $R$ - predicates of:

$$
\Omega=\{\mathrm{U}, \cap, /,+,-, P, C, R\} .
$$

Thus, an algebraic system $\Sigma=(\mathrm{S}, \Omega)$ over a set of program structures and operations on them (union, connection, differences and projections) is defined.

\subsection{The Simple and Complex of Graph Structures Programs}

Among the variety of program structures there are three main ones - a simple, complex structure with a call of modules from the external environment and a dynamic structure. The main purpose of various structures is the most optimal use of the main memory during the execution of the unit (Lavrischeva, 2014, 2017 Yurait).

Simple structure. An aggregate with a simple structure is created in the process of building modules based on the operations of link calls. The amount of main memory occupied by an aggregate with a simple structure is constant and equal to the sum of the volumes of individual modules:

$V_{s}=\sum^{n} v_{i}$, where $v_{i}$ is the amount of memory occupied by the $i$-th module (i=n). The corresponding graph of a modithlar structure is always connected.

Complex structure. Assembly of complex structures with dynamic invocation of modules in the shared memory is created in the Assembly process of the modules. In such an aggregate, the connections between the modules are not so rigid and their sequence is determined by the modules included in the chain. The modules are loaded into the main memory at the time of processing. When finished, the memory is freed and used to load another module. As in the case of a simple structure, the graph of a complex program structure is also connected (Fig.4) and is reflected in the adjacency matrix (2).

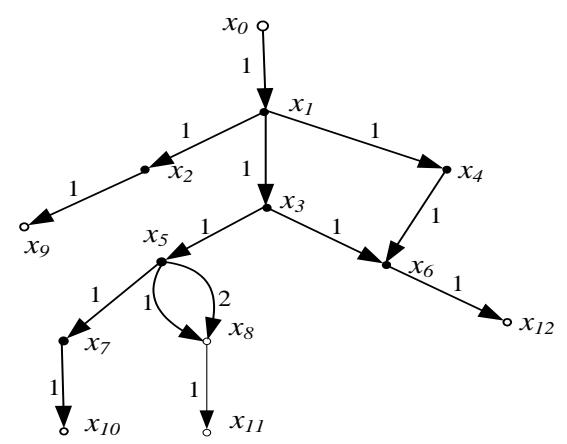

Figure 4. Modification graph of program structure

The amount of main memory required depends on the number and composition of modules and the maximum 
amount of memory is equal to the sum of individual modules:

$$
v_{0}^{\max }=V_{s}=\sum_{i=1}^{n} v_{i}
$$

The minimum amount of memory required when performing the aggregate is calculated by Floyd's algorithm, which determines the shortest path in the graph, in which each arc corresponds to a weight coefficient, called the arc length. The following transformations are performed to apply the Floyd algorithm.

1). Let's add new vertices and arcs to the graph. The vertices are $x_{0}, x_{n+1}, \ldots, x_{n+m}$, where $m$ is the number of end vertices. New arcs include $\quad\left(x_{0}, x_{1}, 1\right),\left(x_{r l}, \mathrm{x}_{\mathrm{n}+1}, 1\right), \ldots,\left(x_{r n}, x_{n+r n}, 1\right)$. In them $x_{1}$ corresponds to the main module and all $x_{i}$ - to the end vertices. After performing operations, the graph of the modular structure (Fig. 1) is given to the graph on Fig. 5 with vertices $x_{0}, x_{9}, x_{10}, x_{11}, x_{12}$. It vertices correspond to the weight coefficients: $v_{0}=v_{9}=v_{10}=v_{11}=v_{12}=0$.

2). Each arc of the form $\left(x_{i}, x_{j}, k\right)$ is assigned a coefficient $v_{i j}=\frac{v_{i}+v_{j}}{2}$.

Consider all routes leading from $x_{0}$ to one of the other additional vertices. The length of the shortest route path is calculated as follows:

$$
l_{0, n+p}=v_{01}+\ldots+v_{r p, n+p}=\frac{v_{0}+v_{1}}{2}+\ldots+\frac{v_{2 p}+v_{n+p}}{2}=\frac{v_{0}}{2}+v_{1}+\ldots+v_{r p}+\frac{v_{n+p}}{2}=v_{1+\ldots}+v_{r p} .
$$

This length $l_{0, n+p}$ will be equal to the sum of the memory modules for path $x_{1}, \ldots, x_{r p}$.

Thus, applying Floyd's algorithm to the graph in Fig. 2, we solve the problem of calculating the amount of memory for the maximum chain.

3). We replace the adjacency matrix with the path matrix. For each $m_{i j}>0$, the corresponding location will be $v_{i j}$. The values $m_{i j}=\varnothing$ are replaced by $-\infty$. The program implementing Floyd's algorithm has the following form (it is assumed that the path matrix is described as a two-dimensional matrix $(n \times n)$ : this length $l_{0, n+p}$ will be equal to the sum of the memory modules for path $x_{1}, \ldots, x_{r p}$.

$$
\begin{aligned}
& \text { for } k=1 \text { to } n \text { do } \\
& \text { for } i=1 \text { to } n \text { do } \\
& \text { for } j=1 \text { to } n \text { do } \\
& \text { if } \mathrm{M}[\mathrm{i}, \mathrm{j}]<\mathrm{M}[\mathrm{i}, \mathrm{k}]+\mathrm{M}[\mathrm{k}, \mathrm{j}] \text { then } \\
& \mathrm{M}[\mathrm{i}, \mathrm{j}]:=\mathrm{M}[\mathrm{i}, \mathrm{k}]+\mathrm{M}[\mathrm{k}, \mathrm{j}] .
\end{aligned}
$$

As a result of this algorithm, a matrix of maximum paths will be constructed. The maximum of $l_{0, n+p}$ will determine the minimum amount of $l_{0, n+p}$ memory for the memory-overlapping aggregate.

The most complex structure for the values $V_{0}{ }^{\min } \leq V_{0} \leq V_{0}^{\max }$ can be constructed by following the algorithms proposed in (Lavrischeva, 2009; Lipaev, 1992). The qualitative dependence of $V_{0}$ on the number of dynamic sites is shown in Fig.5. Here $n$ is the number of modules in the unit. Despite the different kind of curves, they have a common pattern - any $V_{0}$ is enclosed between the values of $v_{0}{ }^{\max }$ и $v_{0}{ }^{\min }$.

Dynamic structure. The mechanism of dynamic links between modules is different from the call mechanism. Dynamic objects are loaded into the main memory when they are accessed. By analogy, we call the volume loaded with a single treatment of a dynamic element, has its own program structure, for which the adjacency matrix is composed. If the same modules are found in different dynamic structures, they are different objects.

The original graph of matrix M is used for illustration (Fig.1). Let the module corresponding to the vertex $x_{1}$, be dynamically called from the module corresponding to the vertex $x_{3}$. The resulting modified graph is shown in Fig. 6 . A dashed arrow indicates a dynamic call. The module corresponding to the vertex $x_{6}$, occurs twice.

We construct an adjacency matrix for this aggregate. Each dynamic element will have its own CALL/RPC. To distinguish a dynamic call, the corresponding matrix elements will contain negative numbers whose absolute values specify the number of dynamic calls between the data of the module pair (Lavrischeva, 2014)..

The adjacency matrix M for Vs as simple strucrure G will look like: 


$$
\mathrm{M}=\left(\begin{array}{ccccccccc}
x_{1} & x_{2} & x_{4} & x_{6} & x_{3} & x_{5} & x_{6} & x_{7} & x_{8} \\
0 & 1 & 1 & 0 & -1 & 0 & 0 & 0 & 0 \\
0 & 0 & 0 & 0 & 0 & 0 & 0 & 0 & 0 \\
0 & 0 & 0 & 1 & 0 & 0 & 0 & 0 & 0 \\
0 & 0 & 0 & 0 & 0 & 0 & 0 & 0 & 0 \\
0 & 0 & 0 & 0 & 0 & 1 & 1 & 0 & 0 \\
0 & 0 & 0 & 0 & 0 & 0 & 0 & 1 & 2 \\
0 & 0 & 0 & 0 & 0 & 0 & 0 & 0 & 0 \\
0 & 0 & 0 & 0 & 0 & 0 & 0 & 0 & 0 \\
0 & 0 & 0 & 0 & 0 & 0 & 0 & 0 & 0
\end{array}\right)
$$

We investigate the qualitative dependence of the amount of the number of dynamic segments (Fig.5. and 6). With one component in the software unit of a simple structure we have $\mathrm{V}_{\mathrm{d}}{ }_{\mathrm{d}}=\mathrm{Vs}$. If each dynamic component consists of one module, then the modified Floyd algorithm finds the maximum path and $\mathrm{V}_{\mathrm{n}}^{\mathrm{d}}=\mathrm{V}_{0}{ }^{\min }$.

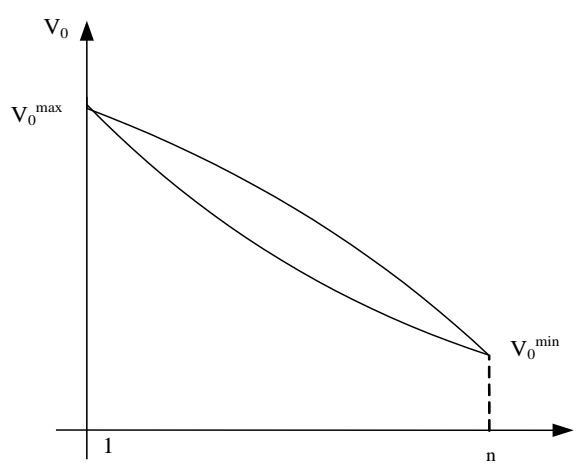

Figure 5. Grafics of qualitative dependence $V_{a}$ from the number of sub graphs

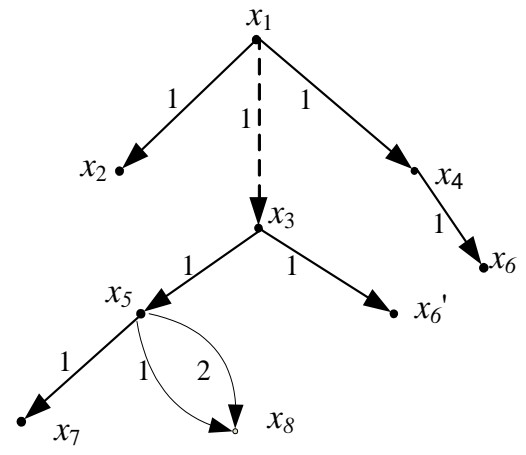

Figure 6. Graph programs structure with dynamic Call's

For intermediate values, the dependence is more complex. On fig.7 presents two curves $(1,2)$, and $n$ is the number of modules in the program unit.

Curve 1 defines a relationship in which different segments do not have the same modules. Curve 2 describes the dependence for the case when different segments have the same modules. For them, the required memory increases due to the duplication of such modules. However, dependence 2 is typical for the case when there are no identical modules in dynamic structures and they are written in high-level $L P$. These modules are handled by utility tools - memory management, I/O, emergency handling, etc.

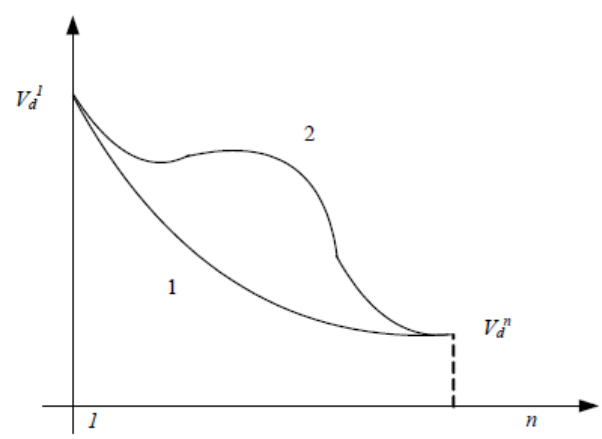

Figure 7. Grafic dependence Va from the number of dynamic elements

Due to the duplication of modules there is an increase in the main memory of the OS. Thus, curve 1 is characteristic of software aggregates of graphs in the form of a tree, which ensures that there are no identical modules in the graph. Despite the lack of dynamic structure in terms of memory savings, there is a significant advantage - independence from editing links. Each dynamic object can be modified, and editing relationships in the $\mathrm{OS}$ is not required. 


\section{Operations of Assembling Programs Elements of Graph}

Let the graph $\mathrm{G}$ be represented by the set of modules $\mathrm{X}=\left\{x_{1}, x_{2}, \ldots, \mathrm{x}_{m}\right\}$, as described in $L P$, and located at the vertices of the graph. The modules are assembled into a software unit. In this case, each pair of modules $x_{i}, x_{j}(i, j$ - languages from the set of $L P$ are connected by the relation of call on the basis of which the module of communication $\mathrm{x}_{i j}^{\prime}$ is formed. In General, for simple program structures, the aggregate contains link communication (call) operators and forward and reverse transformations of data types passed from the calling module (in $i$-language) to the calling module (in $j$-language) and back (Lavrischeva, 2014, 2016, 2017).

After 1992 it are for link equivalent operations: make BSD, Java (1996); assembling, config SPAROL, Grid (2002), etc. (Lavrischeva, 2017, 2018 OS Day; 2018 Jsea). Then there were standard languages of the description of interfaces of IDL, API, WSDL and the statement of config of the standard IEEE 828-2012 (Configuration Management) for receiving a configuration file of any AS from modules, objects, components, services and other the ready resources in new LP.

LP allows you to describe the information part - passport modules with a description of the transmitted data (Lavrischeva, 2016; 2016, 27December; 2018 jsea) and operations call modules. Taking into account the passports of the modules, the software structure of the unit is built (program - Prog, complex - Comp, package $P a c)$. The passport describes the special language WSDL containing: a subset of the operations associate link elements of the graph in the language $L^{\prime}$ that contains a description of the parameters from the list of actual and formal parameters of the invocation; mathematical operations on the graph and operations of linking modules. The operator modules link (make, config, assembling, etc) takes the general form:

link <aggregate type> <aggregate name> (<main module name>, <additional list of module names>) <execution mode>, when constructing specific program structures, the vertices of the graph - modules can be marked with special symbols $\rho$, denoting:

$\rho=\alpha$ formation of a fragment with the name of the module;

$\rho=*$ the beginning of the dynamic fragment with the vertex marked by this symbol;

$\rho=+$ the module in the graph $G$ is marked as the main program of the complex;

$\rho=/$ means enabling debugging or testing of the unit.

Using these designations, the graph $\mathrm{G}$ will take the form shown in figure 8 and has a representation:

$$
E=\left\{\left(x_{5}, x_{7}, 1\right),\left(x_{5}, x_{8}, 1\right),\left(x_{5}, x_{8}, 2\right)\right\} .
$$

The aggregate is given a unique name corresponding to the generated root module. For the graph $\mathrm{E}=\left\{\left(x_{4}, x_{6}, 1\right)\right\}$ a fragment of operators providing a dynamic call will be formed in the communication module $x_{46}^{\prime}$. For a pair of modules specified in Fig. 8 vertices $x_{4}, x_{6}$, the structure of the corresponding part of the unit, including the communication module, is shown in Fig. 9. Similarly links of heterogeneous modules and other types of calls are implemented.

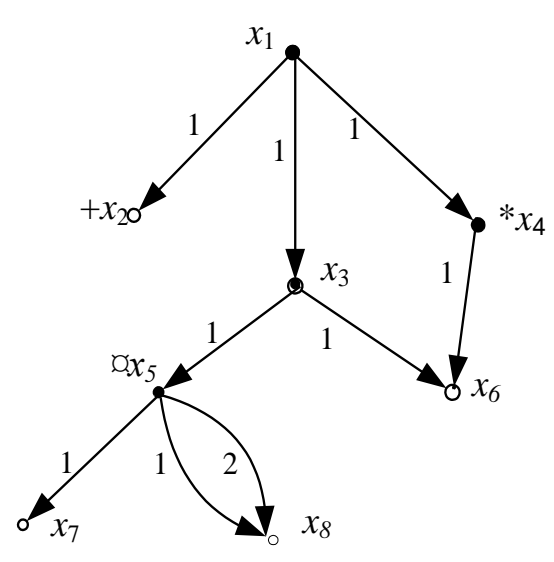

Figure 8. Graph of software unit with control marks on the graph

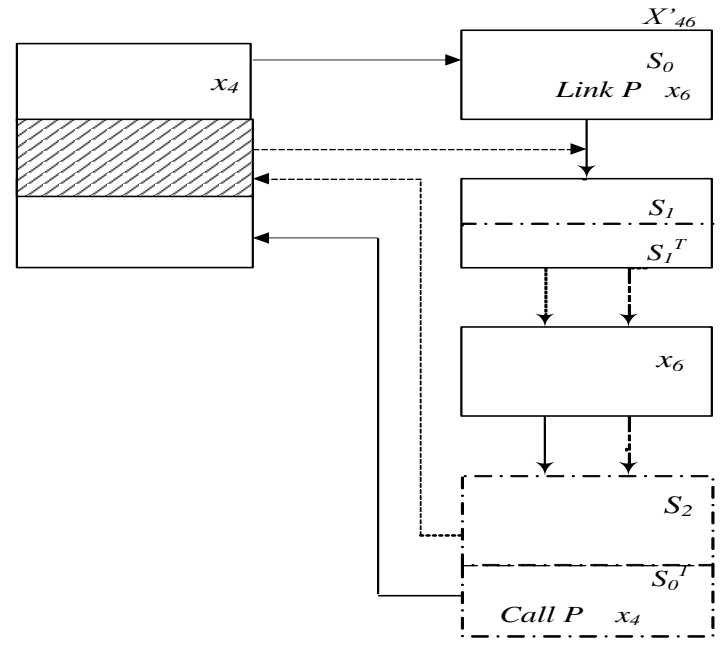

Figure 9. Graph of modular structure with dynamic calls (Lavrischeva, 1982) 
Thus, for a pair of modules $\mathrm{x}_{i}, \mathrm{x}_{\mathrm{j}}$, a module of connection $\mathrm{x}_{\mathrm{ij}}$ of the form:

$$
x_{i j}^{\prime}=S_{0} *\left(S_{1} \times S_{1}^{T}\right) *\left(S_{2} \times S_{2}^{T}\right) * S_{0}{ }^{l},
$$

where $S_{0}$ is a fragment of the aggregate that defines the environment of $x j$ module functioning;

$\mathrm{S}_{1}$ - a fragment of the aggregate, including a sequence of calls to functions from the set $\{P, C, S\}$, each of which performs the necessary conversion of the actual parameters when referring to the $\mathrm{x}_{\mathrm{j}}$-module;

$\mathrm{S}_{2}-$ a system with a fragment of operators for the inverse transformation of data types transmitted from $x_{j}$ to $x_{i}$ after its execution;

$\mathrm{S}_{0}{ }^{l}-\mathrm{a}$ piece of software structures with operators epilogue for the vertex $x_{i}$, for the restoration of the environment.

For the described program structures, set the link operations to build the individual programs in Fig.8:

$$
\begin{aligned}
& \operatorname{Link} \operatorname{Prog} \mathrm{P}_{1}\left(\mathrm{x}_{1}, \mathrm{x}_{2}\right) ; \\
& \operatorname{Link} \operatorname{Prog} \mathrm{P}_{2}\left(\mathrm{x}_{1}, \mathrm{x}_{3}\right)\left(\mathrm{x}_{3}, \mathrm{x}_{6}\right) ; \\
& \operatorname{Link} \operatorname{Comp} \mathrm{P}_{3}\left(\left(\mathrm{x}_{1}, \mathrm{x}_{3}\right)\left(\mathrm{x}_{3}, \mathrm{x}_{5} / x_{58}^{\prime}\right)+\left(\mathrm{x}_{5}, \mathrm{x}_{7}\right) ;\right. \\
& \operatorname{Link} \operatorname{Prog} \mathrm{P}_{4}\left(\mathrm{x}_{1}, \mathrm{x}_{4}\right),\left(\mathrm{x}_{4}, \mathrm{x}_{6}\right) ; \\
& \operatorname{Link} \operatorname{Comp}\left(P_{1} \mathrm{U} P_{2} \mathrm{U} P_{3} \mathrm{U} P_{4}\right)
\end{aligned}
$$

Programs of the complex (aggregate) are given unique names $\left(\mathrm{P}_{1}, \mathrm{P}_{2}, \mathrm{P}_{3}, \mathrm{P}_{4}\right)$ corresponding to the root names of the modules in the chains of the graph.

Thus, the process of constructing the program structure on the graph includes:

1. Enter the module description in the $L P\left(L^{\prime}\right)$ and perform syntax checking.

2. Select the required modules and interfaces from the repositories and place them in the graph.

3. Translation of the unit modules in the $L P$.

4. Generation of communication modules for each interconnected pair of graph modules.

5. Assembly of the elements of the graph in the finished structure, linking modules in the operating system (IBM, MS, Oberon, Unix и др.).

6. Test the system on data sets and assess the reliability of the unit.

After the modules are built, the name of the software Assembly is entered into the boot library. If you create a fragment that is later included in another aggregate, its name must match the name of the main module. In connection with the transition to the Internet environment to work with various software and system services in the configuration assembly of such tools provides security, data protection and quality assessment of ready-made modules, service resources and web systems in Internet (Lavrischeva, 2018 Abrau).

\subsection{Assembling Theory of the Programs Modules to Systems}

Method based on the interface that specifies the modules to each other and exchanging data (1975-1982). Interface - intermodule, interlanguage and technological (Interface-SEV, 1987).

The intermodule interface is a module proxy (stub, skeleton) between two communicating modules to exchange data. Interlanguage interface defines the methods convert the transferred data types of PL using algebraic systems and 64 functions of the library interface in APROP. The formal conversion of (TD) objects of the Assembly is performed using algebraic systems for each $t$-boolean-b; character-c; integer-I; real-r; and array-a, union-u, record-r (Lavrischeva, 2014, 2016, 2017, 2018 jsea).

$$
\begin{gathered}
T_{\alpha}{ }^{t}: \quad G_{\alpha}{ }^{t}=\left\langle X_{\alpha}{ }^{t}, \Omega_{\alpha}{ }^{t}\right\rangle, \text { where } \\
t \text { - the data type } b, c, i, r, a, z, u, r, e ; \\
X_{\alpha}{ }^{t}-\text { a set of values for variables of that type; } \\
\Omega_{\alpha}{ }^{t} \quad \text { - a set of operations on these TD. }
\end{gathered}
$$

For simple and complex TD modern PL built classes of algebraic systems:

$$
\begin{aligned}
& \Sigma_{1}=\left\{G_{\alpha}{ }^{b}, G_{\alpha}{ }^{c}, G_{\alpha}{ }^{i}, G_{\alpha}{ }^{r}\right\}, \\
& \Sigma_{2}=\left\{G_{\alpha}{ }^{a}, G_{\alpha}{ }^{2}, G_{\alpha}{ }^{u}, G_{\alpha}{ }^{e}\right\} .
\end{aligned}
$$

Systems $\Sigma_{1}$ and $\Sigma_{2}$ the transformation $t \rightarrow q$ for the pair of languages $L_{t}$ and $L_{q}$ have the properties: 
1) $G_{\alpha}{ }^{t}$ and $G_{\beta}{ }^{q}$ - isomorphic to $\mathrm{q}$ and t defined on the same set;

2) $X_{\alpha}{ }^{t}$ and $X_{\beta}{ }^{q}$ are isomorphic if $\Omega_{\alpha}{ }^{t}$ and $\Omega_{\beta}{ }^{q}$ are different. If $\Omega=\Omega_{\alpha}{ }^{t} \cap \Omega_{\beta}{ }^{q}$ is not empty, then there is a isomorphism between $\mathrm{G}_{\alpha}{ }^{t^{\prime}}=\left\langle\mathrm{X}_{\alpha}{ }^{t}, \Omega>_{\text {и }} \mathrm{G}_{\beta}{ }^{q^{\prime}}=\left\langle\mathrm{X}_{\beta}{ }^{q}, \Omega\right\rangle\right.$.

3) Between the sets $X_{\alpha}{ }^{t}$ and $X_{\beta}{ }^{q} \quad$ may not be isomorphic matching, then build such a mapping between $X_{\alpha}{ }^{t}$ and $X_{\beta}{ }^{q}$ that it is isomorphic.

Theorem. Let $\varphi$ - displays the algebraic system $\mathrm{G}_{\alpha}{ }^{c}$ to $\mathrm{G}_{\beta}{ }^{c}$. In order to $\varphi$ be an isomorphism, it is necessary and sufficient to $\varphi$ isomorphic reflected $X_{\alpha}^{c}$ and $X_{\beta}^{c}$, preserving linear order.

Each class of systems transformation $t \rightarrow q$ for the pair of languages $l q$ and $l t$ of PL may be some properties of mappings:

- systems $G_{\alpha}{ }^{t}$ and $G_{\beta}{ }^{q}$ are isomorphic if their $q, t$ are defined on the same set TD;

- between the values of $X_{\alpha}^{t}$ and $X_{\beta}^{q}$ of data types $t, q$ there is an isomorphism if the set of operations $\Omega_{\alpha}{ }^{t}$ and $\Omega_{\beta}{ }^{q}$ are different;

- if the set $\Omega=\Omega_{\alpha}{ }^{t} \cap \Omega_{\beta}{ }^{q}$ is not empty, then we have the isomorphism of the two systems $\mathrm{G}_{\alpha}{ }^{{ }^{\prime}}=<G_{\alpha}{ }^{t^{t}}$ $=\left\langle X_{\alpha}{ }^{t}, \Omega\right\rangle$ и $G_{\beta}{ }^{q^{\prime}}=\left\langle X_{\beta}{ }^{q}, \Omega\right\rangle$.

If data types are different, for example, $t$ - string, and type $q$ is real, then there is no isomorphic correspondence between sets $X_{\alpha}^{t}$ и $X_{\beta}^{q}$.

The maps preserve the linear order of the elements based on the linear order of the elements of the

algebraic systems of these classes.

These methods put on the beginning assemble programming in USSR (Lavrischeva, 1982, 1991; Lipaev, 1992).

\section{Operations assembling after 1990}

Languages for describing object links in different environments it was such: make GNU, BSD, Java (1996); config, building, assembling Grid (2002), etc. (Lavrischeva, 2009, 2014, 2017). The make statement by RPC/RMI provides Assembly of executable modules from Filemake libraries and sets them the order of links with each other in Linux, BSD, GNU, Java, Grid and other (http://xrnlrpc.scripting.corn/spec.html).

Operators config, building, assembling worked out in the European Grid project. Further development of the Assembly was factory programs (D. Greenfield and K. Lenz - stream Assembly 2007; K, A. Chernetsky and Azinaker - multy-conveyor 2005; I. Bay - the interaction of multi-language programs in distributed environments, 2005; R. Pohle - conveyor Assembly for Product Line/Product Family, 2004, etc.).

The theory of factories on a method of Assembly for production of programs from ready reuses with processes of planning, management, estimation of cost and quality of a product was formed. In 2012, the Assembly (integration) of ready-made reuses was standardized in IEEE 828-96, 2012 (Configuration Management) to obtain the configuration file of any application system from ready-made resources (Lavrischeva, 2015 London; 2017 29-30 November; 2017 IEEE).

\subsection{Ready-Made Software Elements (Reuses) Configuration to the System}

Under the configuration of the system is understood the structure of some of its version, including software elements, combined with each other by link operations with parameters that specify the options for the functioning of the system (Lavrischeva, 2018 jsea; 2018 Abrau). Version or variant of system configuration according to the IEEE Standard 828-2012 (Configuration) includes:

- configuration basis (BC);

- Configuration items;

- program elements (modules, components, services, etc.) included in the graph;

Configuration Management is to monitor the modification of configuration parameters and components of the system, as well as to conduct system monitoring, accounting and auditing of the system, maintaining the integrity and its performance. According to the standard, the configuration includes the following tasks:

1. Configuration identification.

2. Configuration Control.

3. Configuration Status Accounting. 
4. Configuration audit.

5. Trace configuration changes during system maintenance and operation;

6. Verification of the configuration elements (components) and testing of the system.

A configuration build uses a system model and a set of out-of the-box components that accumulate in the operating environment repositories or libraries, and selects their operating environment Configurator (for example, in http://etics.cern.ch/eticsPortal, http://7dragons.ru/ru). The configurator assembles the components according to their interfaces and generates the system configuration file.

The Configurator assembly of components and reuses with operation config, which is equivalent to the operations link (20) for figure 8, taking into account their interfaces. The config statement generates a program variant or system configuration file of AS.

\subsection{Quality Assurance of Systems from Modules}

One of the main conditions for the implementation of the Assembly method is to ensure the safety and quality of individual elements and software and hardware systems as a whole. The international Committee of the ACM, IEEE, etc. has created a number of standards to ensure the quality, reliability and certification for different types of systems (ISO 9126, 9000(1-4), 10005: 1995 - 2000; 14598: 1998-2010: GOST 51901-2002, etc.). The ISO/IEC 12207 life cycle standard has been supplemented by the processes of planning, quality management and certification of the system.

These processes are used to analyze the achievement of quality; verification and validation (V\&V) of resources and assessment of the degree of achievement of individual quality indicators; testing of the finished system; data collection on failures, defects and other errors; reliability measurement by predictive, evaluation and measurement models. based on the results of testing (Andon, 2007; lavrischeva, Pakulin, 2018, http://0x1.tv/20180517F).

As an example, consider the ISO/IEC 9000 (1-4) Quality model. It defined six quality indicators: q1 functionality, q2 - reliability, q3 - usability, q4 - efficiency, q5 - maintainability, q6 - portability. To assess the reliability index q2 used 3 kinds of metrics (external, internal and operational) and testing data on failures and defects through the appropriate reliability models (Musa, Morando, Zelinskogo, Verall, etc.). The measure of quality is determined by the formula:

$$
Q_{n c}=\sum_{i=1}^{k} a_{i} \cdot R_{i}
$$

where $a_{i}$ is a measure of the weight, importance of the $i$-th function of the system $(i=k)$ for the process, $R_{i}$ is the reliability of the $\mathrm{i}$ - functions in the period $t$ of the system operation.

Data for all indicators of quality (q-quality) $\mathrm{q}_{1}-\mathrm{q}_{6} \mathrm{c}$ is estimated taking into account the reliability

$\mathrm{R}_{\mathrm{i}}\left(\mathrm{q}_{\mathrm{i}}=2\right)$ according to the formula:

$$
q_{1}=\sum_{j=1}^{6} a_{1 j} m_{1 j} w_{1 j}
$$

where $\mathrm{AI}$ are the attributes of each indicator of quality $(\mathrm{i}=1-6) ; \mathrm{m}_{\mathrm{ij}}$ - metric $\mathrm{i}$-metric with $\mathrm{j}$-values of the quality attributes; wij - weight of i-index with $\mathrm{j}$ - weights the quality of the system. Based on the data collected during the system test, the formula is substituted with the corresponding values of these metrics for each quality indicator. The obtained data on the quality model indicators are included in the product quality certificate (Lipaev, 1982, 2010; Lavrischeva, 2018 17-18 October, http://0x1.tv/20180517F).

\section{The Modern and Future Paradigm Programming}

Paradigm (from Greek. $\pi \alpha \rho \alpha ́ \delta \varepsilon \imath \gamma \mu \alpha$, "model, pattern") - a set of fundamental scientific attitudes, the concepts and terms adopted and shared by the scientific community. Provider continuity of development of science and scientific creativity. Thomas Kuhn called paradigms established systems of scientific views, in which research and development.

In Software Engineering emerged programming paradigm. It is a set of ideas, concepts, theories and methods that determine the style of formal presentation of computer programs. This term R. M. Floyd defined in his work 
"The Paradigms of Programming" (Communications of the ACM. 1969. V. 22 (8). P. 455-460) and E. Dijkstra in the book "Discipline of programming" (M.: Mir, 1976) And D. Gris in the book "Science of programming". Paradigms it is a method of conceptualization and formal definition of programs and systems. Some parts of the theory are implemented in the framework of applied (functional, logical, automatic, etc.), theoretical (VDM, OOP, Z, B, OCM, FODA, etc.), system types (parallel, distributed, etc.) and commercial (Agile, SCRUM, EX) programming (Lavrischeva, 2017 Yurait).

\subsection{Theoretical Programming Paradigms}

Theory of graphs for design software modular structures with mathematical operations (union, projection, difference, etc.) implementation of linking the graph modules (objects) and the semantics of the transformation of data transmitted by the vertices of the graph G (Lavrischeva, 1991, 2014, 2018 jsea). .

\section{Object-component methods - OCM}

$O C M$ are the mathematical design of systems from ready-made resources (objects, components, services, etc.) to OM (Object Model). It is the formal method which transform the elements $O M$ to a component model or a service model (Lavrischeva, 2016 OCM, 2018 ISP , http://0x1.tv/20181122AF ).

Graph objects is designed on four levels:

Generalizing for determining $S D$ base notions without considering of their essences and properties;

Structuring for ordering objects in the $O M$ taking into account relationships between them;

Characterization for forming concepts of objects on the base of them properties and descriptions;

Behavioral level for descriptions of conduct depending on events (such as time).

That is, vertices of the graph $G$ are objects of two types: $O=\left(O_{0}, O_{1}, O_{2} . O_{n}\right)$ with the object relations hold $\forall i(i>0) \Rightarrow\left(O_{i} \in O_{0}\right)$ and interface objects $I$ (Fig.10).
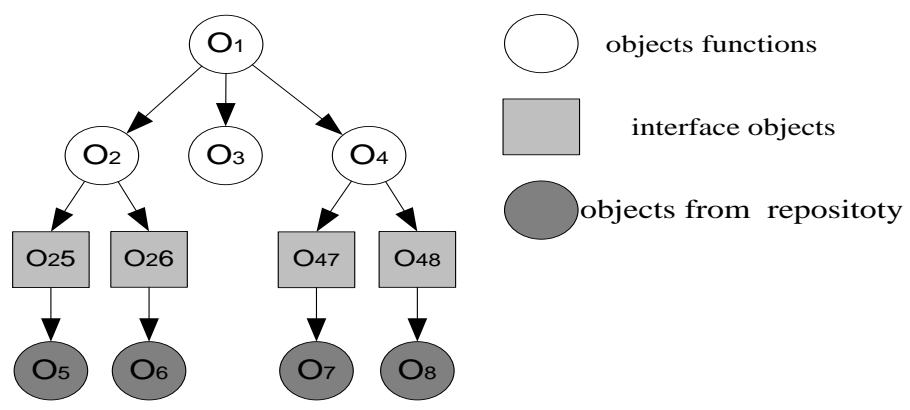

Figure 10. Object-interface graph G

At the vertices of a graph $G$ contains the functional objects $O_{1}, O_{2}, O_{3}, O_{4}, O_{5}, O_{6}, O_{7}, O_{8}$ and interface objects $-\mathrm{O}_{25}, \mathrm{O}_{26}, \mathrm{O}_{47}, \mathrm{O}_{48}$, which are placed in the repository of system, and arcs correspond to relationships between all kinds of objects (Lavrischeva, 2014 jsea, 2016). The parameters of the external characteristics of the interface objects are passed between objects through specified interfaces and are designated in language IDL in (input interface), out (output) and inout (intermediate). Based on the graph $G$ we can construct a program $P_{0}$ $-P_{5}$ using mathematical operation $\cup$ Assembly link:

1) $P_{0}=\left(P_{1} \cup P_{2} \cup P_{3} \cup P_{4} \cup P_{5}\right)$.

2) $P_{1}=O_{2} \cup O_{5}$, link $P_{1}=\operatorname{In} O{ }^{\prime}{ }_{5}\left(O_{2} \cup O_{5}\right)$;

3) $\mathrm{P}_{2}=\mathrm{O}_{2} \cup \mathrm{O}_{6}$, link $\mathrm{P}_{2}=\operatorname{In} \mathrm{O}_{6}^{\prime}\left(\mathrm{O}_{2} \cup \mathrm{O}_{8}\right)$;

4) $P_{3}$;

5) $P_{4}=O_{4} \cup O_{7}$, link $P_{4}=\operatorname{In} O_{7}^{\prime}\left(O_{4} \cup O_{7}\right)$;

6) $\mathrm{P}_{5}=\mathrm{O}_{4} \cup \mathrm{O}_{8}$, link $\mathrm{P}_{4}=\mathrm{In} \mathrm{O}_{8}^{\prime}\left(\mathrm{O}_{4} \cup \mathrm{O}_{8}\right)$;

The set of objects and interfaces of the graph is reflected by general or individual properties and descriptions of the object model. Verification of properties of objects is provided by the specific operations (classification, 
specialization, aggregation, etc.).

Component paradigm. The basis of this paradigm - OCM graph in which vertexes are the components of the CRP (reuses), interfaces and arcs specify the subject classification and the relationship between the vertices. Components are described by the formalisms of the triangle of Frege (Lavrischeva, 2014jsea, 2016, 2018 IJANS).

- sign - identifier of the real function entity;

- denotation - the designation of this entity;

- concept - a set of properties defined by logical connections and must be true. Operations of OCM and component algebra represented on the website http://7dragons/ru/ru (in the VS environment - MS, IBMSphere, Java, Linux, Intel etc.).

Service-component paradigm. System and service-components - web resources implement intellectual knowledge of specialists about applied fields in the Internet environment (Lavrischeva, 2017). Each implements some function and communicates with the technological interface to interact with other services through protocols and provide Assembly and solution of applications of different nature. The means of describing the application systems include:

XML for description and construction of SSA components;

WSDL to describe web services and their interfaces;

SOAP to determine the formats of requests to the web services;

UDDI for integration of services and their storage in libraries;

Configuration (config) of the service resources in some high-quality and secure.

The theory of graphs develop in the school of A.P. Ershov (V.I. Kasyanov, V.E. Itkin, A. A. Evstigneev et al.) for programming Systems. The graph theory has been actively developing in the Russian Academy of Sciences (Lavrischeva, 2017; http://0x1.tv/20181122AF). The theory of conformity for systems with blocking and destruction for the schematic organization of memory in Linux.

Methods of production of factories (Product Line/Product Family) programs and Appfab and certificate them of the quality are discussed (Lavrischeva, 2011, 2013 com, 2014, 2017).

Application of the ontology language OWL (www.semantic_web.com), resource language (RDF) and intelligent agents of ISO 15926 standard for networking.

Ontology of Life Cycle and Computational geometry is a part of computer graphics and algebra. Used in the practice of computing and control machines, numerical control etc. is also used in robotics (motion planning and pattern recognition tasks), geographic information systems (geometric search, route planning), design chips, etc. (Lavrischeva, 2014, 2015, 2017 Yurayt).

Cloud technologies (PaaS, SaaS) are related to the Internet and are used to create adaptive applications that interact through agents of web pages (Lavrischeva, 2014, 2017, 2018).

Device configuring Big Data Processing Devices (Big Data) in Smart Data Internet 4.0 (Lavrischeva, 2016, 2018).

\subsection{Application Technical Programming}

Event management paradigm based on the processing of external events (event-driven programming) in the Window environment. Features of the event paradigm are the use of testing methods based on operational (scenario) profiles of programs (Andon, 2007).

Coordinated and parallel programming provides a division of the computational process into several subtasks (processes) for TRAN's computers and supercomputers, the results of which are sent via communication channels. Languages for parallel programming - PVM, LAM. CHMP and MPI (Message Passing Interface) interface descriptions and OpenMP. The POSIX standard provides messaging between programs in LP of C, C+ and Fortran.

Programming on classes and on a prototype in OOP. The principles of the OOP are:

inheritance - the mechanism of establishing relations "descendant-ancestor" (the ability to generate one class from another with the preservation of all the properties and methods of the class-ancestor); encapsulation (the hiding of class implementation); abstraction (description of interaction only in terms of messages/events in the 
subject area); polymorphism (the possibility of replacing the interaction of objects of one object with another object with a similar structure). Many modern languages are specially created for programming on classes, for example, Smalltalk, C++, Java, Python, PHP, Object Pascal (Delphi), VB.NET, Xbase, etc.

Programming by prototype. Creating a new object is done by one of two methods: cloning an existing object, or by creating an object from scratch. Reuse (inheritance) is made by cloning an existing instance of the object -a prototype Clone, a sample. An example of a prototype language is the Self language and it is the basis of such programming languages as JavaScript, Squeak, Cecil, Newton Script, Io, MOO, REBOL, Keno and etc.

The Agile methodology is focused on the close collaboration of a team of developers and users. It is based on a waterfall model lifecycle incremental and rapid response to changing demands on PP. The team works according to the schedule and financing of the project (Andon, 2007).

eXtreme Programming (XP) implements the principle of "collective code ownership". It any member of the group can change not only your code but also code another programmer. Each module is supplied with the Autonomous test (unit test) for regression testing of modules. Tests written by the programmers and they have the right to write tests for any module. Thus, most of the errors are corrected at the stage of encoding, or when you view the code, or by dynamic testing.

SCRUM is agile methodology project management firm Advanced Development Methods, Inc., used in organizations (Fuji-Xerox, Canon, Honda, NEC, Epson, Brother, 3M, Xerox and Hewlett - Packard etc.) are based on an iterative lifecycle model with well-defined development process, including requirements analysis, design, programming, testing (http://agile.csc.ncsu.edu).

DSDM (Dynamic Systems Development Method) for rapid development of RAD (Rapid Application.

\subsection{Perspective Directions for the Development of the Internet}

Promising areas of development for the Internet1 include (Lavrischeva, 29-30 November 2017). The information objects (IO) that specifies the digital projection of real or abstract objects that use Semantic Web Ontology interoperability interfaces. IO through Web services began more than 10 years ago. Interaction semantics IO is based on RDF and OWL language of ISO 15926 Internet 3.0.

The next step of the development of the Internet is Web 4.0, which allows network participants to communicate, using intelligent agents. A new stage in the development of enterprise solutions-cloud (PaaS, SaaS) who spliced with Internet space and used to create Adaptive applications. Cloud services interact through the Web page by using agents.

Internet of Things Smart IoT to support competitive APPS using: distributed microservices; Hypercat Mobile; GSM-R traffic control. Industrial Internet develops concepts - "smart energy", "smart transportation", "smart appliances", "smart industry", "smart homes and cities", etc. Internet stuff (Internet of Things, Smart IoT) indicates the Smart support competing APPS using distributed micro services such as Hyper cat (mobile communications); industrial Internet (Industrial), covering the new automation concepts-smart energy, transportation, appliances, industry», and another.

\subsection{Computer Nanotechnologies}

Today computer nanotechnology is actually already working with the smallest elements, "atoms" similar to the thickness of the thread (transistors, chips, crystals, etc.). For example, a video card from 3.5 million particles on single crystal, multi-touch maps for retinal embedded in the eyeglasses, etc.

In the future, ready-made software elements will be developed in the direction of nanotechnology by "reducing" to look even smaller particles with predetermined functionality. Automation of communication, synthesis of such particles will give a new small element, which will be used like a chip in a small device for use in AS (Lavrischeva, 29-30 November).

\section{Conclusion}

In the early stages of the emergence of the method of assembling large programs and complexes of spent modules in the LP used the theoretical apparatus of graphs to create modular program structures. Graph theory allows to establish the shortest path of program elements and prove the correctness of binding graph modules using adjacency matrices, reachability and mathematical operations (association, connection, etc.) in complex program structures (complex, aggregate, system, etc.). Initially, the method of Assembly on the basis of graph theory was widely implemented in the Ruza systems, Prometheus Complex under the leadership of V.V.Lipaev and was supported by A. P. Ershov in the IPI SO Academy of Sciences USSR and his researcher and scientist, who formulated the theoretical aspects of the application of graph theory in programming. Since 2013, graph 
theory has been used in the modeling of complex systems of objects, components, services by OCM and has been used in the world practice in the transformation data to the Internet environment (Lavrischeva, 2018 Abrau). This was carried out in the framework of the project RFBR №16-000-00352 under the modeling complex systems graph structures from services resources which configuration (config) by Standard 828-2012. Elements of the graph set transition labels to obtain reactions at the time of exposure to test sets and proof of completeness of testing systems of AS and ensure their quality (Lavrischeva, 2018 17-18 October; 2018 ISP). New developed programming paradigms for the period up to 50 years of the 21 st century are justified as perspective directions of development of the graph theory of modeling of complex systems from heterogeneous software and intellectual resources for vital areas of society (medicine, biology, physics, mathematics, etc.).

\section{Reference}

Andon, F. I., \& Koval, G. I. et al. (2007). Fundamentals of software systems quality engineering. K.: Nauk. Dumka, 2007. 670 p. In Rus.

Bruno, C., \& Joost, E. (2016). Graph structure and monadic second-order logic. A language- theoretical approach (hal id: hal-oo646514).

Burdonov, I. B., Kosachev, A. S., \& Kulyamin, V. V. (2008). Theory for systems with locks and destructions. Moscow (411p.). In Rus.

Ekaterina, L., Andrey, S., \& Andriy, K. (2014, jsea). Object-Component Development of Application and Systems. Theory and Practice. Journal of Software Engineering and Applications, 2014. Retrieved from http://www.scirp.org/journal/jsea

Ekaterina, M. L. (2016). Assemblling Paradigms of Programming in Software Engineering. (pp. 296-317). https://doi.org/10.4236/jsea.2016.96021

Ershov, A. P. (1977). Introduction to the theory of programming. Moscow (287p.). In Rus.

Evstigneev, A. N. (1985). Graph theory in programming. Moscow, Nauka (351p.). In Rus.

Glushkov, V. M., Stogniy, A. A., \& Lavrischeva, E. M. et al. (1976). Automation System production of programs (APROP). Kiev. IK AN USSR (134p.). In Rus.

Halstead, M. H. (1981). The beginnings of a science about the programs. Perevod. with ang. M.: Finance and Statistics (201p.). In Rus.

Horn, E., \& Winkler, F. (1987). Design of modular structures. Computer technology of the socialist countries, 21, 64-72.

Kotov, V. E. (1978). Introduction to the theory of program schemes. Novosibirsk, SB of AS USSR (173p.) In.Rus.

Koval, G. I., Korotun, T. M., \& Lavrischeva, E. M. (1987). On one approach to solving the problem of intermodule and technological interface. All the collection of the Academy of Sciences and Min.Education of the USSR (pp.52-68). In Rus.

Lavrischeva, E. (2015). Ontological Approach to the Formal Specification of the Standard Life Cycle. Science and Information Conference-2015, Jule 28-30, London, UK, p.965-972. https://doi.org/10.1109/SAI.2015.7237259

Lavrischeva, E. M. (2014). Software Engineering of computer systems. Paradigms, technologies, CASE- means Programming, Kiev: Nauk Dumka (284p.). In Rus.

Lavrischeva, E. M. (2016). The theory of object-component modeling of software systems. Preprint of the RAS, No. 29 ( 48 p.). ISBN 078-5-91474-025-9.13.

Lavrischeva, E. M. (2017). Software engineering and programming technology of complex systems. Textbook. 2nd edition, Moscow, Yurayt. 428p.

Lavrischeva, E. M. (2017, IEEE). Development of the theory programs and systems in the USSR History and modern Theory. Sorucom, 2017, IEEE Springer 2017. p.31-47. https://doi.org/10.1109/SoRuCom.2017.00011

Lavrischeva, E. M. (2018). The Scientific basis of software engineering. International Journal of Applied and Natural Sciences (IJANS), 7(5), 15-32.

Lavrischeva, E. M., \& Grishchenko, V. N. (1982). The connection of multi-language modules in the OS of the ES. Moscow Finance and statistics, 127. In Rus. 
Lavrischeva, E. M., \& Grishchenko, V. N. (1991). Assembly programming. Kiev: Nauk. Dumka (136p.). In Rus.

Lavrischeva, E. M., \& Grishchenko, V. N. (2009). Assembly programming. Basics of software industry products, Kiev. Nauk. Dumka (371p.).In Rus.

Lavrischeva, E. M., \& Petrenko, A. K. (2018, ISP). Informatics -70. computerization aspects of programming software and informatic systems technologies. ISP RAS. Proc., 29(5), 7-30. https://doi.org/10.15514/ISPRAS-2018-30(5)-1

Lavrischeva, E. M., \& Ryzhov, A. G. (2016). Application the theory of General data types of ISO/IEC 11404 GDT standard in relation to Big Data. Paper presented at the conference "Actual problems in science and ways their development". Retrieved from http://euroasia-science.ru (pp. 99-110). In Rus.

Lavrischeva, E. M., \& Ryzhov, A. G. (2018, Abray). Approach to modeling of systems and sites from ready-made resources. Scientific service on the Internet: proceedings of the XX All-Russian scientific onference (17-22 September 2018g. Novorossiysk). IPM im. M. V. Keldysh. p. 321-345. https://doi.org/10.20948/abrau-2018-50

Lavrischeva, E. M., Mytulyn, V. S., Kozin, S. V., \& Ryzhov, A. G. (2017). Creation of the application and Information Systems from ready-made Internet resources. The proceedings of ISP RAS. M., 30(1), 27-40.

Lavrischeva, E. M., Pakulin, N. V., Ryjov, A. G., \& Zelenov, S. V. (2018). Analysis of methods of Assessment reliability of equipment and systems. Practice of application of methods of reliability.-Scientific-practical conference - OS DAY, Moscow. The proceedings of ISP RAS, 30(3), 99-120. https://doi.org/10.15514/ISPRAS-2018-30(3)-8

Lavrischeva, K. A., \& Aronov, A. D. (2013). Programs Factory - A conception of Knowledge Representation of Scientific Artifacts From Standpoint of Software Engineering. Comp. and Inf. Sci., 21-28.

Lavrischeva, K. M. (2011). Theoty and Practice of Software Factories. Cybernetic and Systems Analyses, 47(6). 961-972. https://doi.org/10.1007/s10559-011-9376-5

Lavrischeva, E. M. (2018). Scientific Foundation of the System Programming. Journal of Software Engineering And Applications (JSEA), 11(8), 408-434. https://doi.org/10.4236/jsea.2018.118025.

Lavrishcheva, E. M., \& Petrov, I. B. (2017, 29-30 November). Ways of Development of Computer Technologies to Perspective Nano. Future Technologies Conference (FTC), 29-30 November 2017, Vancouver, Canada, p.540-549.

Lipaev, V. V. (2014). Software engineering of complex custom software products.-Textbook. Moscow-2014, Max-Pres., 308p. In Rus.

Lipaev, V. V., Posin, B. A., \& Shtrik, A. A. (1992). The Technology of Assembly programming. Moscow (284 p.). In Rus.

\section{Copyrights}

Copyright for this article is retained by the author(s), with first publication rights granted to the journal.

This is an open-access article distributed under the terms and conditions of the Creative Commons Attribution license (http://creativecommons.org/licenses/by/4.0/). 